\title{
ON THE $p$-REDUCED ENERGY OF A GRAPH
}

\author{
MIRJANA LAZIĆ
}

\begin{abstract}
Let $G$ be a simple connected graph of order $\mathrm{n}$ and let $\lambda_{1} \geq \lambda_{2} \geq$ $\cdots \geq \lambda_{n}$ be the spectrum of $G$. Then the sum $S_{k}^{l}(G)=\left|\lambda_{k+1}\right|+\left|\lambda_{k+2}\right|+\cdots+$ $\left|\lambda_{n-l}\right|$ is called $(k, l)$-reduced energy of $G$, where $k, l$ are two fixed nonnegative integers [2]. In this work, we make a generalization of the $(k, l)$-reduced energy, as follows: for any fixed $p \in N$, the sum $S_{k}^{l}(G, p)=\left|\lambda_{k+1}\right|^{p}+\left|\lambda_{k+2}\right|^{p}+\cdots+$ $\left|\lambda_{n-l}\right|^{p}$ is called the $p$-th $(k, l)$-reduced energy of the graph $G$. We also here introduce definitions of some other kinds of the $p$-reduced energies and we prove some properties of them.
\end{abstract}

\section{INTRODUCTION}

In this paper we consider only simple connected graphs. The vertex set of a graph $\mathrm{G}$ is denoted by $V(G)$, and its order by $|G|$. The spectrum of such a graph is the set $\lambda_{1} \geq \lambda_{2} \geq \cdots \geq \lambda_{n}$ of eigenvalues of its 0-1 adjacency matrix [1].

Let $N_{0}$ be the set of all nonnegative integers and $l \in N_{0}$ be a fixed number. For any graph $G$ with $|G|=n>l$ the sum of eigenvalues $\left|\lambda_{1}\right|+\left|\lambda_{2}\right|+\cdots+\left|\lambda_{n-l}\right|$ is denoted by $S_{+}^{l}(G)$ and is called $l$-positive reduced energy of $G[2]$. In this work we shall define the sum $S_{+}^{l}(G, p)=\left|\lambda_{1}\right|^{p}+\left|\lambda_{2}\right|^{p}+\cdots+\left|\lambda_{n-l}\right|^{p}$ which is called the $p$-th $l$-positive reduced energy of the graph $G$. It contains at least the largest eigenvalue $\lambda_{1}(G)$ of $G$. We note that $\left|\lambda_{1}\right| \geq 1$, hence $S_{+}^{l}(G, p) \geq 1$ for any graph $G$. For any real $a \geq 1$ and any $l \in N_{0}$ and $p \in N$, we can consider the class of graphs $E_{+}^{l}(p, a)=\left\{G: S_{+}^{l}(G, p) \leq a\right\}$.

Now, we prove an important property of the general class $E_{+}^{l}(p, a)$.

Theorem 1. For every constant $a \geq 1$ and for any fixed $l \in N_{0}$ and $p \in N$, the class of connected graphs $E_{+}^{l}(p, a)=\left\{G:\left|\lambda_{1}\right|^{p}+\cdots+\left|\lambda_{n-l}\right|^{p} \leq a\right\}$ is finite.

Proof. Let $a$ be any real number $(a \geq 1)$ and $l$ be nonnegative integer. Let $G$ be a graph of order $n>l$ from the class $E_{+}^{l}(p, a)$. Then

$$
S_{+}^{l}(G, p)=\left|\lambda_{1}\right|^{p}+\cdots+\left|\lambda_{n-l}\right|^{p} \leq a,
$$

2000 Mathematics Subject Classification. Primary: 05C50.

Key words and phrases. Simple graphs, Energy of the graph. 
which provides that

$$
\begin{aligned}
\sum_{\left|\lambda_{i}\right| \geq 1}\left|\lambda_{i}\right|^{p} & \leq \sum_{i=1}^{n-l}\left|\lambda_{i}\right|^{p}+\sum_{i=n-l+1}^{n}\left|\lambda_{i}\right|^{p} \leq \\
& \leq a+\sum_{i=n-l+1}^{n}\left|\lambda_{1}\right|^{p}=a+l \cdot\left|\lambda_{1}\right|^{p} \leq a(l+1)
\end{aligned}
$$

Relations (1) and (2) now give

$$
2(n-1) \leq 2 m=\sum_{i=1}^{n}\left|\lambda_{i}\right|^{2} \leq \sum_{\left|\lambda_{i}\right|<1}\left|\lambda_{i}\right|+\sum_{\left|\lambda_{i}\right| \geq 1}\left|\lambda_{i}\right|^{p} \leq(n-1) \cdot 1+a(l+1)
$$

where $m$ is the number of edges of the graph $G$. Using (3) we find that $n \leq$ $a(l+1)+1$, which completes the proof.

Next, let $k, l \in N_{0}$ and $p \in N$ be any fixed numbers. For any graph $G$ with $|G|=n>k+l$, the sum of eigenvalues $\left|\lambda_{k+1}\right|+\left|\lambda_{k+2}\right|+\cdots+\left|\lambda_{n-l}\right|$ is denoted by $S_{k}^{l}$ and is called $(k, l)$-reduced energy of $G$. The sum of $p$-th degrees of eigenvalues $\left|\lambda_{k+1}\right|^{p}+\left|\lambda_{k+2}\right|^{p}+\cdots+\left|\lambda_{n-l}\right|^{p}$ is denoted by $S_{k}^{l}(G, p)$ and is called the $p$-th $(k, l)$ reduced energy of the graph $G$. For any real $a>0$, for any $k, l \in N_{0}$, and for $p \in N$, we can consider the class of graphs

$$
E_{k}^{l}(p, a)=\left\{G: S_{k}^{l}(G, p) \leq a\right\} .
$$

We note that the $p$-th $(0, k)$-reduced energy of $G$ is $p$-th $k$-positive reduced energy of $G$. We can always assume that $k, l \in N_{0}$ and $p \in N$.

Since the complete bipartite graph $K_{m}^{n}$ belongs to the class $E_{k}^{l}(p, a)$ for any $m, n \in N$, the class $E_{k}^{l}(p, a)$ is always infinite. In what follows, we will prove an important property of this kind of energy on the set so-called canonical graphs.

We say that two vertices $x, y \in V(G)$ are equivalent in $G$ and write $x \sim y$ if $x$ is non-adjacent to $y$, and $x$ and $y$ have exactly the same neighbors in $G$. Relation $\sim$ is an equivalence relation on the vertex set $V(G)$. The corresponding quotient graph is denoted by $\widetilde{G}$, and is called the canonical graph of $G$.

Consequently, for any real $a>0$ and $k, l, p \in N$, we can consider the class of the corresponding canonical graphs

$\tilde{E}_{k}^{l}(p, a)=\left\{G: G \in E_{k}^{l}(p, a)\right.$ is a canonical graph $\}$.

If $k=l$, then $S_{k}^{k}(p, a), E_{k}^{k}(p, a), \tilde{E}_{k}^{k}(p, a)$ are simply denoted by $S_{k}(p, a)$, $E_{k}(p, a)$ and $\tilde{E}_{k}(p, a)$, respectively.

We now prove an important property of the class $\tilde{E}_{k}(p, a) \quad(a>0, k, p \in N)$. It is based on two theorems proved in [3].

Theorem 2. For every constant $a>0$ and any positive integers $k, p \in N$, the class of connected graphs $\tilde{E}_{k}(p, a)$ is finite.

Proof. On the contrary, we shall suppose that for some $a>0$ (a is a positive integer) and $k, p \in N$ the set $\tilde{E}_{k}(p, a)$ is infinite. By Theorem proved in [3], for 
any real number $A>0$, there exists a graph $G \in \tilde{E}_{k}(p, a)$, which has $q>A$ nonzero eigenvalues. This graph will satisfy the relation

$$
\left|\lambda_{k+1}\right|^{p}+\left|\lambda_{k+2}\right|^{p}+\cdots+\left|\lambda_{n-k}\right|^{p} \leq a .
$$

Suppose that $\lambda_{r}>\lambda_{r+1}=\cdots=\lambda_{r+s}=0>\lambda_{r+s+1}$, where $s=n-q$ is the multiplicity of zero of this graph. The characteristic polynomial of $G$ is then

$$
P_{n}(\lambda)=\lambda^{s}\left(\lambda^{q}+a_{1} \lambda^{q-1}+\cdots+a_{q}\right),
$$

where $\left|a_{q}\right|=\lambda_{1} \cdots \lambda_{r} \cdot\left|\lambda_{r+s+1}\right| \cdots\left|\lambda_{n}\right|$.

By Theorem also proved in [3], we shall suppose that $s=0$ and thus $n=q$. Also, we can assume that $n$ is so large that we have $\sqrt{n} \geq a+6 k$.

It is clear that $\left|\lambda_{i}\right| \leq n-1$ for $i \in\{1,2, \ldots, k\} \cup\{n-k+1, n-k+2, \ldots, n\}$, and $\left|\lambda_{i}\right| \leq \sqrt{n}$ for $i=k+1, k+2, \ldots, n-k$.

We can assign with $t$ the total number of eigenvalues $\lambda_{i}$, with $\left|\lambda_{i}\right| \leq 1 / \sqrt{n}$ $(i=k+1, k+2, \ldots, n-k)$. It is easy to see that $t>a+4 k$. On the contrary, we would have that there exist at least $n-(2 k+t)$ eigenvalues $\lambda_{i}(k+1 \leq i \leq n-k)$ with $\left|\lambda_{i}\right|>1 / \sqrt{n}$. By relation (4) we have

$$
a \geq \sum_{i=k+1}^{n-k}\left|\lambda_{i}\right|^{p}>\frac{n-(2 k+t)}{\sqrt{n}}>\sqrt{n}-\frac{a+6 k}{\sqrt{n}} .
$$

From relation $\sqrt{n} \geq a+6 k$ and relation (5) we have $a>\sqrt{n}-1$ what is a contradiction.

Denote with $t_{0}$ the total number of all eigenvalues $\lambda_{i}(i=k+1, k+2, \ldots, n-k)$ with $\left|\lambda_{i}\right|>1$. By relation (4) we have

$$
a \geq\left|\lambda_{k+1}\right|^{p}+\cdots+\left|\lambda_{n-k}\right|^{p} \geq \sum_{\left|\lambda_{i}\right|>1}\left|\lambda_{i}\right|^{p} \geq t_{0}
$$

which provides that $t_{0} \leq a$.

We now have

$$
\begin{aligned}
\left|a_{n}\right| & =\left(\left|\lambda_{1}\right| \cdots\left|\lambda_{k}\right|\right)\left(\left|\lambda_{k+1}\right| \cdots\left|\lambda_{n-k}\right|\right)\left(\left|\lambda_{n-k+1}\right| \cdots\left|\lambda_{n}\right|\right) \leq \\
& \leq(n-1)^{2 k} \underbrace{\sqrt{n} \cdot \sqrt{n} \cdots \sqrt{n}}_{t_{0}} \cdot \underbrace{\frac{1}{\sqrt{n}} \cdot \frac{1}{\sqrt{n}} \cdots \frac{1}{\sqrt{n}}}_{t} \underbrace{1 \cdot 1 \cdots 1}_{n-\left(t+t_{0}+2 k\right)}<1,
\end{aligned}
$$

which is a contradiction $\left(\left|a_{n}\right| \in N \quad a_{n} \neq 0\right)$. Consequently, the set $\tilde{E}_{k}(p, a)$ is finite for every $a>0$ and every $k, p \in N$.

Corollary 1. For every constant $a>0$ and any positive integers $k, l, p \in N$, the class of connected graphs $\tilde{E}_{k}^{l}(p, a)$ is finite.

Proof. Without loss of generality, we can assume that $k \geq l$. Let $G$ be any graph from the class $\tilde{E}_{k}^{l}(p, a)$. Since

$$
a \geq \sum_{i=k+1}^{n-l}\left|\lambda_{i}\right|^{p}=\sum_{i=k+1}^{n-k}\left|\lambda_{i}\right|^{p}+\sum_{i=n-k+1}^{n-l}\left|\lambda_{i}\right|^{p} \geq \sum_{i=k+1}^{n-k}\left|\lambda_{i}\right|^{p}
$$


we have $G \in \tilde{E}_{k}(p, a)$, thus $\tilde{E}_{k}^{l}(p, a) \subseteq \tilde{E}_{k}(p, a)$. Since the class $\tilde{E}_{k}(p, a)$ is finite for every $a>0$ and every $k, p \in N$, we get the statement.

\section{REFERENCES}

[1] D.Cvetković, M.Doob and H.Sachs, Spectra of Graphs-Theory and Application, Deutscher Verlag der Wissenschaften, Berlin and Academic Press, NewYork, 1982.

[2] M.Lepović, Some kinds of energies of graphs, Discrete Mathematics 128(1994), 277-282.

[3] A.Torgašev, On infinite graphs with three and four non-zero eigenvalues, Bull. Serb. Acad. Sci. et Arts LXIV (Sci. Math.) 11(1981), 39-48.

FACUlTy OF SCIENCE

InstituTE OF MATHEMATICS AND INFORMATICS

P.O.Box 60, Radoja Domanovića 12

34000 Kragujevac

SERBia AND Montenegro

E-mail address: mmmvl@kg.ac.yu 